\title{
NEW BAT LOCALITY RECORDS FROM CUBA WITH EMPHASIS ON THE PROVINCE OF MATANZAS
}

\author{
Nuevas localidades y registros de murciélagos para Cuba, \\ con énfasis en la provincia de Matanzas
}

\author{
Johanset Orihuela $^{1 *}$, Lázaro W. Viñola ${ }^{2}$, and Ricardo A. Viera ${ }^{3}$ \\ ${ }^{1}$ Earth and Environment (Geosciences), Florida International University, Miami, Florida, 33199, USA; \\ (D) orcid.org/0000-0002-7806-603X ; *Para correspondencia: jorih003@fiu.edu. ${ }^{2}$ Florida Museum of Natural History, \\ University of Florida, Gainesville, FL 32611-7800; (Dorcid.org/0000-0002-2956-6759. ${ }^{3}$ Progressus Heritage and \\ Community Foundation, USA; (D) orcid.org/0000-0002-6535-1472.
}

\section{ABSTRACT}

Here we provide a compilation of bat distribution records based on neontological and paleontological data, updating the known distribution in Matanzas province, the Isle of Pines, and Central Cuba. From 97 collecting localities in the Province of Matanzas, we report 27 taxa out of the 34 known from the Cuban archipelago; 21 of them are extant while the other six are extinct. Antrozous koopmani and Natalus primus are considered locally extinct in Matanzas, as in most of the archipelago today, but had a wider distribution in the past that lasted until very late in the Holocene. The extinct endemics, Artibeus anthonyi, and Phyllops vetus, are reported for the first time in the province, and the distribution records of Phyllops falcatus, Lasiurus pfeifferi, Lasiurus insularis, Chilonatalus macer, and Eumops ferox are updated and expanded. These records make Matanzas the second richest province in bat diversity of the Cuban archipelago and an area of considerable conservation potential.

Keywords: bats, Matanzas, fossils, Cuba, extinction, biogeography.

\section{RESUMEN}

Se hace una actualización del registro de murciélagos para la provincia de Matanzas, Isla de Pinos y zona central de Cuba, basada en reportes paleontológicos y neontológicos de un total de 97 localidades de colecta. Para la provincia de Matanzas reportamos 27 especies de 34 registradas, entre extinguidas y vivientes, representando un $79 \%$ de la quiropterofauna conocida y más del $84 \%$ de las especies vivientes del archipiélago cubano. Las especies en alto riesgo de extinción Antrozous koopmani y Natalus primus, aunque localmente extintas en la provincia, como en la gran mayoría de la isla, presentaron un registro fósil que sugiere una mayor distribución hasta el Holoceno tardío. Las especies extinguidas endemicas Artibeus anthonyi y Phyllops vetus se reportan por vez primera para Matanzas. Además, se actualizan y expanden los registros de Phyllops falcatus, Lasiururs pfeifferi, Lasiurus insularis, Chilonatalus macer y Eumops ferox. Las nuevas localidades y registros hacen de la región de Matanzas la segunda provincia de mayor diversidad quiropterologica del archipiélago cubano, distinguiéndose como un área de considerable potencial para la conservación de los murciélagos de Cuba. 


\section{INTRODUCTION}

The Cuban archipelago is a center of bat biodiversity in the Antilles. The main island of Cuba, including the Isle of Pines and surrounding keys, have the greatest bat richness with 27 living species, seven of them endemics, representing nearly half (45\%) of the recorded species of the entire Antillean sub-region (Silva Taboada, 1979; Morgan \& Wood, 1986; Morgan, 2001; Mancina \& García, 2011; Mancina, 2012). Of this fauna, 16 species (60\%) utilize caves for roosting or diurnal refuge, of which $10(37 \%)$ are considered strict cave-dwellers. Fewer species are arboreal (Silva Taboada, 1979; Mancina, 2012). In addition to the current diversity, there are seven species (excluding the extinct M. magna) of which two, Mormoops megalophylla and Desmodus rotundus, are extirpated. So far, four species are considered accidental records: Eumops perotis, Lasiurus cinereus, Myotis sodalis and Ariteus flavescens (Gundlach, 1868; Silva Taboada, 1979; see Balseiro, 2011, for an alternative interpretation).

Identifying sites or areas of high species richness and providing inventories of their diversity are among the most important aspects of conservation (Longino \& Colwell, 1997; Sánchez-Lozada et al., 2018). Since Gilberto Silva Taboada's work on Cuban bats (1979), the chiropteran fauna within the region of Matanzas, among others in the archipelago, has been partially updated or reassessed. The most recent have concentrated on providing inventories from National Parks or Park Reserves, which have nevertheless provided new locality records throughout the archipelago (Díaz-Franco, 2005; Mancina, 2011, 2012; Mancina \& García, 2011; García \& Mancina, 2011; Balseiro, 2011; Borroto-Páez \& Mancina, 2017; Sánchez-Lozada et al., 2018). However, most of these recent compilations did not include paleontological data or subfossil/fossil-derived records, which we consider important for the understanding of former fauna diversity and changes in local distribution; both which are imperative for long-term conservation planning. Recent paleontological data have already hinted at the region's rich historical diversity (Viera, 2004; Viera \& Orihuela, 2006; Orihuela, 2011, 2010, 2013; Orihuela \& Tejedor, 2012), but these have not been assimilated into some of the current distributional compilations or inventories (García \& Mancina, 2011; Mancina, 2012; Sánchez-Lozada et al., 2018).

\section{OBJECTIVES}

- Our goal here is to present a species-location compilation based on paleontological and neontological records available since the XIX century, including 41 new localities and observations that are absent from previous inventories or species distribution research.

\section{MATERIALS AND METHODS}

The records are based on observations, sample collections, and paleontological work executed between 1995 and 2018. Identification and systematic taxonomy follow Silva Taboada (1976, 1979), Silva Taboada and Vela (2009) and Mancina (2012). We considered Antrozous koopmani as an endemic species (Silva Taboada, 1976; Silva Taboada \& Vela, 2009), although it has been considered as a subspecies of the continental form $A$. pallidus (Simmons, 2005; Fig. 2). The extinct Mormoops magna is here tentatively considered conspecific with Mormoops megalophylla, and Desmodus puntajudensis is considered a full synonym of Desmodus rotundus (Orihuela, 2010, 2011). 
Species abbreviations. The species roster is organized alphabetically based on taxonomic abbreviations as follows: Artibeus anthonyi (AA), Artibeus jamaicensis parvipes (Ajp), Antrozous koopmani (Ak), Brachyphylla nana (Bn), Chilonatalus macer (Chm), Desmodus rotundus (DR), Eumops ferox (Ef), Eptesicus fuscus dutertreus (Efd), Erophylla sezekorni (Es), Lasiurus insularis (Li), Lasiurus pfeifferi (Lpf), Mormoops blainvillii (Mb), Mormoops megalophylla (MM), Molossus molossus tropidorhynchus (Mmt), Monophyllus redmani clinedaphus (Mrc), Macrotus waterhousiei minor (Mwm), Nycticeius cubanus (Nc), Noctilio leporinus mastivus (Nlm), Natalus primus (Npr), Nyctiellus lepidus (Nyl), Phyllops falcatus (Pf), Phyllonycteris poeyi (Pp) and Phyllops vetus (PV). Taxa abbreviations and valid subspecies follow Silva Taboada (1979) and Tejedor (2011). Validity follows Simmons (2005). Subspecies were included only for the purpose of creating the abreviations, and discussed in the text where necessary. All capitals indicate extinct/extirpated species. The subspecies pertain to those recognized for Cuban species (Silva Taboada, 1979). An asterisk (*) indicates species reported only from fossil or subfossil material. Only species that were irrefutably identified were counted in our records. Uncertain records, such as Nyctinomops macrotis from Cueva de la Caja (Viera, 2004) or a live specimen of Antrozous koopmani from the region of Guamuhaya (see Mancina, 2012) are not counted here, largely because these specimens were not preserved or lack any other form of record and are thus not available for study or confirmation. Species referred and illustrated here were donated to the Museo Nacional de Historia Natural, La Habana, Cuba, but not all have been cataloged.

Rarity indices were calculated to represent abundance-collection frequency following Silva's Taboada (1979) equation $=\sqrt{\mathrm{AF} / 10}$; where $\mathbf{A}$ stands for specimens resulting from collection or observation, and $\mathbf{F}$ stands for the total number of collecting stations from which the $\mathbf{A}$ collection originated. The resulting score was arbitrary organized as follows: very common $(>15$ collection localities), common (11-15), scarce (6-10) and rare $(<5)$.

Localities. The collection or sampling localities are organized in occurrence (Fig. 1). Our locality list includes a compilation of previous reports (Gundlach, 1868; Chapman, 1892; Miller, 1904; Allen, 1911, 1917, 1918., Anthony, 1917; Barbour, 1945; Aguayo, 1950; Silva Taboada, 1974, 1976, 1979; Garrido, 1980; Mancina et al., 2004; Tejedor et al., 2004, 2005; Viera, 2004; Silva Taboada \& Vela, 2005; Orihuela, 2010, 2011; García \& Mancina, 2011; Hernández Muñoz \& Mancina, 2011; Tejedor, 2011; Valdés et al., 2011; Mancina, 2012; Orihuela \& Tejedor, 2012; Borroto-Páez \& Mancina, 2017; Sánchez-Lozada et al., 2018).

Thus providing the perspective of over a century of records (Tables I-II). Altitudes are approximate given in meters above present sea level taken from Google Earth Pro (2019). Current vegetation and original vegetation, when discussed, are from personal observations, the Cuban National Atlas (Instituto de Planificación Física, 1989), González et al. (2012) and Borroto-Páez and Mancina (2017). The gazetteer of localities is provided in Table I. 

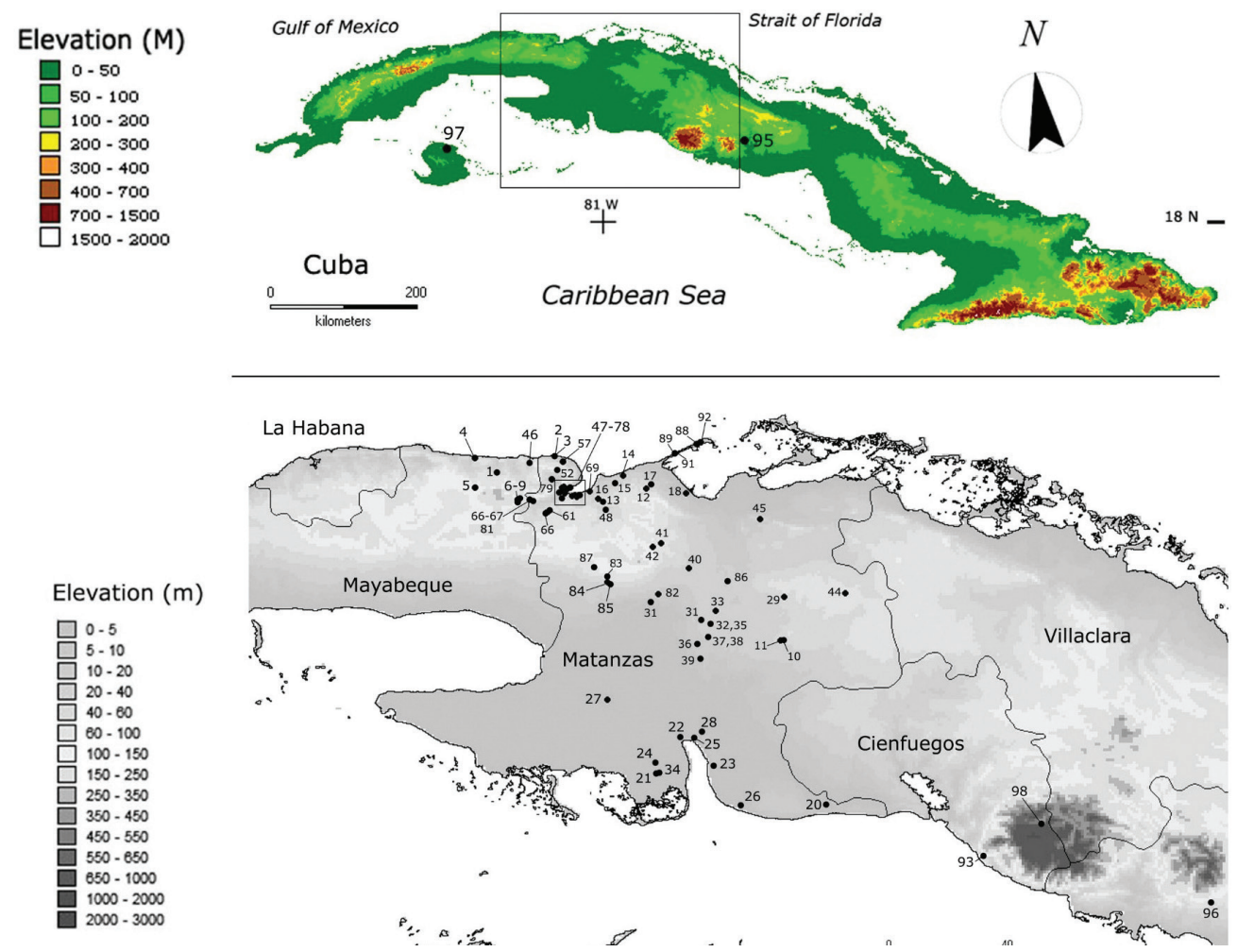

Figure 1. Distribution of sampled localities and bat collecting stations in Cuba (location number are indexed on Table I database).
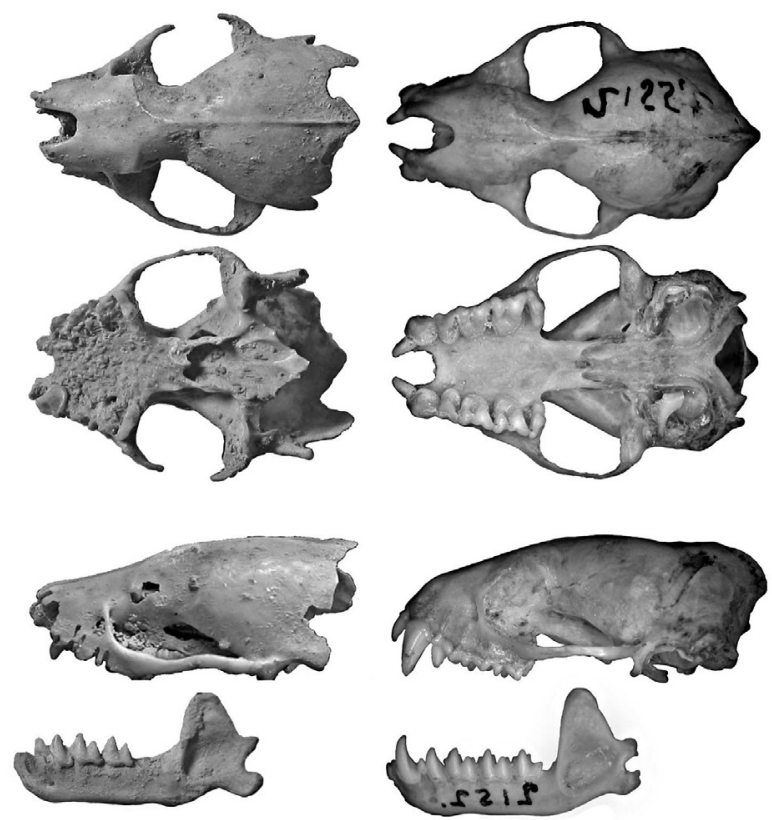

$10 \mathrm{~mm}$

Figure 2. Comparison between the endemic Cuban Pallid bat (Antrozous koopmani) from Cueva de los Nesofontes (loc. 6) and the North American Pallid bat (Antrozous pallidus, AMNH 2152 mandible, and the skull AMNH 2155, both females from Guadalajara, Mexico). 

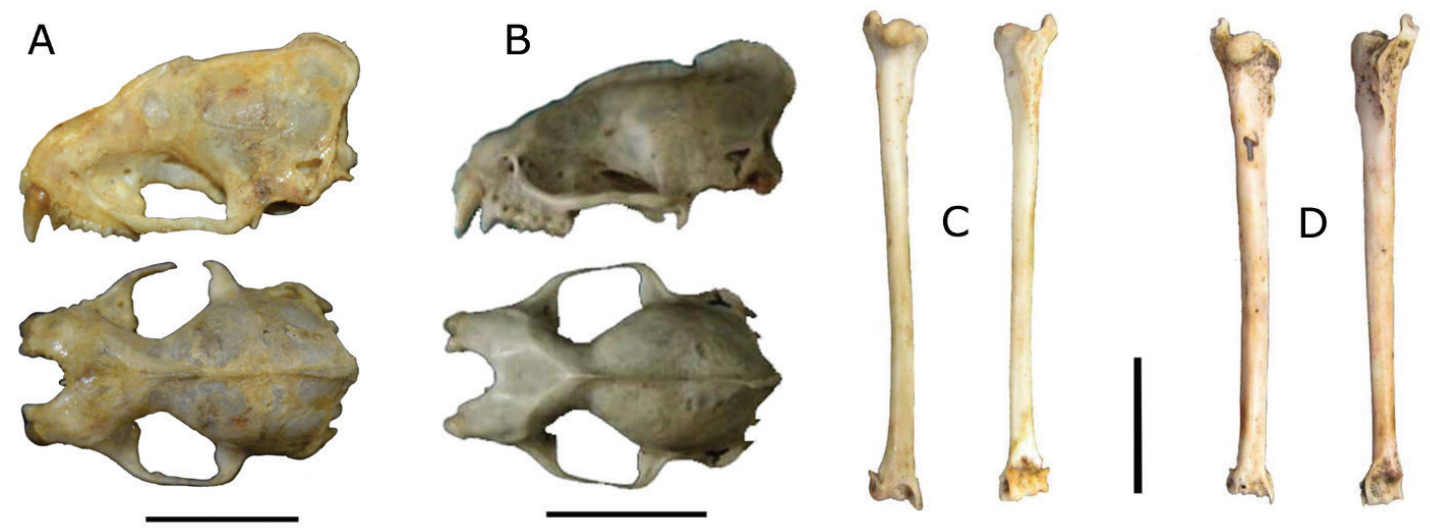

Figure 3. Neontological Lasiurus insularis and Eumops ferox specimens. A, Lasiurus insularis extracted skull from specimen collected and reported by Viera (2004) from the Yumurí River Gorge, in the outskirts of Matanzas city (locality 77). B, Lasiurus insularis skull and humerus, C, from a modern owl roost at J4-Quarry (locality 38). D, Eumops ferox from Hornos de Cal site (locality 95) in Santi Spíritus. All scale bars $=10 \mathrm{~mm}$.
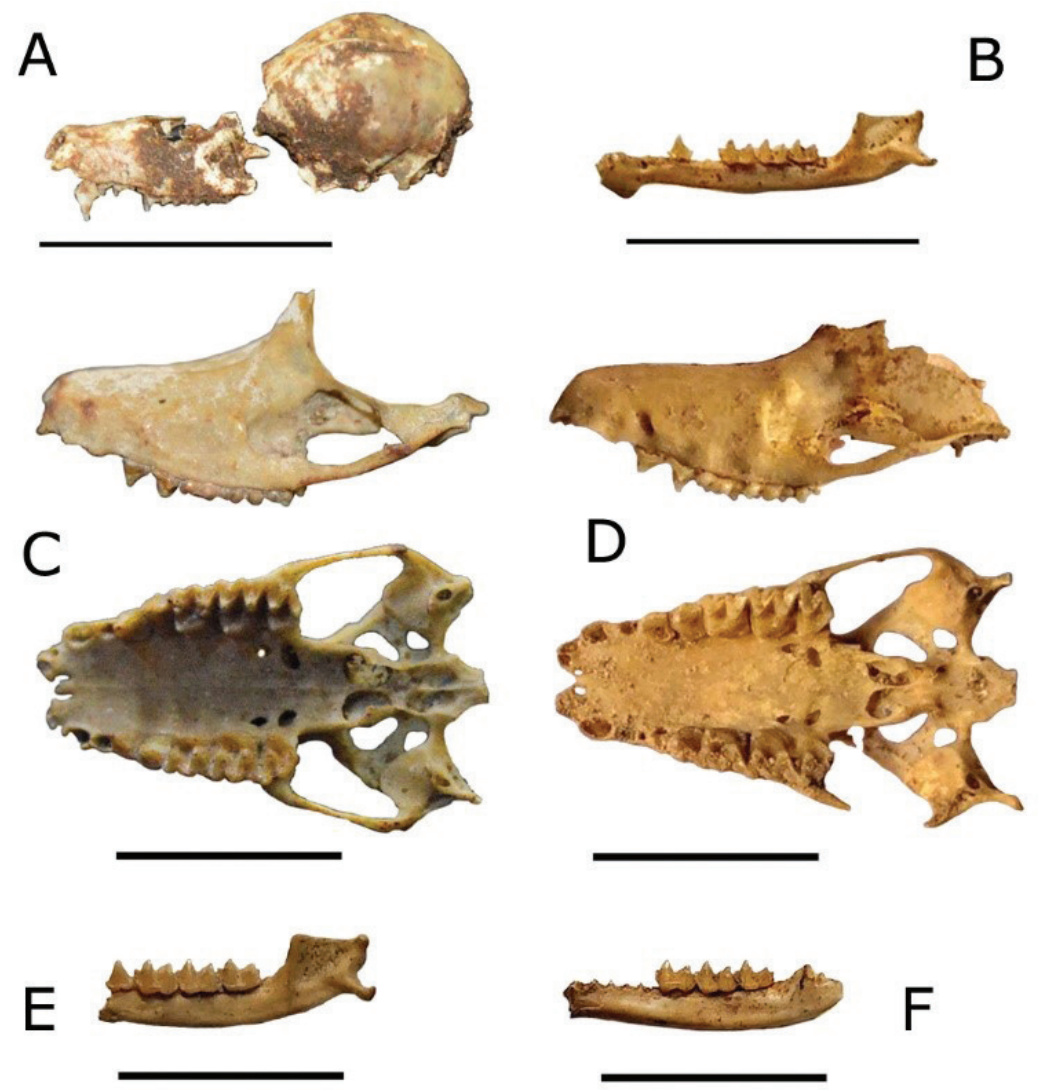

Figure 4. Very fresh natalids from two owl roost deposits in Matanzas. A, Chilonatalus macer fragmentary skull from Cueva del Ciclón. B, Chilonatalus macer left dentary from a Tyto furcata fresh pellet in a modern roost at Cueva del Ciclón (Loc. 62). C-E, Natalus primus rostra and fragment of a left dentary from the same locality and in association to Ch. macer from above. F, Natalus primus right dentary (image mirrored) from Cueva del Gato Jíbaro (loc. 58). All scale bars $=10 \mathrm{~mm}$. 


\section{RESULTS}

Here we report a total of 27 bat species in 19 genera, including extant and extinct taxa, distributed throughout 97 localities including the Sierra de Caballos in the Isle of Pines (Isla de Pinos), Cienfuegos, Villa Clara, and Santi Spíritus, but with emphasis on the Province of Matanzas (Table I). Of the 26-living species registered for the Cuban archipelago, we report 21 taxa from the region of Matanzas alone, representing $84 \%$ of the extant bat diversity of the Cuban archipelago.

In the region of Matanzas, 41 new localities are added to the existing record, including both modern and fossil/subfossil specimens. At the same time, we increase the record of species from several of the already published localities. Among these, ocurrences in owl pellets or from cave localities and urban centers comprise $55.7 \%$ and $22.7 \%$ of the collection localities respectively; and together over $78 \%$ of the total localities. Rural localities constituted $21.6 \%$ of all localities, but are comparable to urban localities (Table I-II).

Of the overall total of 27 recorded species, four represent extinct forms: Artibeus anthonyi, Desmodus rotundus, Mormoops megalophylla, and Phyllops vetus, which constitute $57 \%$ of the known extinct and extirpated species from Cuba. Artibeus anthonyi and Phyllops vetus constitute new comfirmed fossil records for the region of Matanzas. Phyllops vetus is also recorded for the first time in Villa Clara province. Several other records now extend the modern distributions of Lasiurus insularis, Lasiurus pfefferi, Chilonatalus macer and Eumops ferox (Figs. 3-4; Table I). An additional record of Lasiurus insularis is provided from modern owl roost at the J4 quarry (loc. 37), plus two modern records of Eumops ferox from which it was previously unrecorded: one captured inside a house in the city of Cárdenas (loc. 17, by RV) and another from a fresh owl pellet from Cueva del Indio 2 (by LWV, loc. 66; Fig. 3D).

The critically endangered and very rare species Antrozous koopmani and Natalus primus are reported from paleontological deposits only, although some of them are possibly very recent in age (Fig. 2). These species had already been registered from fossil, subfossil and neontological records by several authors across the island (Silva Taboada, 1976, 1979; Viera, 2004; Orihuela, 2011, 2010; Tejedor, 2011; Orihuela \&Tejedor, 2012, Mancina, 2012). The modern (i.e., post-Columbian) specimens of Natalus primus were discovered in what seemed to be "fresh" owl pellets in modern owl roosts in Cueva del Gato Jíbaro and Cueva Ciclón (Fig. 4), two caves of the Bellamar system near the city of Matanzas. A specimen under similar conditions had been previously reported from Cueva Ciclón by Viera (2004) and from Gato Jíbaro by Tejedor (2011). Fossil remains of Phyllops falcatus are reported for the first time from Cueva Musulmanes, Varadero peninsula, suggesting the presence of this species there at least in the Holocene. This last specimen is highly mineralized and likely does not have enough collagen for radiocarbon dating. 


\begin{tabular}{|c|c|c|c|c|c|c|c|c|c|c|c|c|c|c|c|c|c|c|c|c|c|c|c|c|c|c|}
\hline हิ & & & & $\star$ & *: & & $\rtimes$ & & $n$ & & & & $n$ & $\approx$ & & & & & & & & & $n$ & $\approx \times$ & & \\
\hline$\vec{z}$ & & & & & 薺 & & & & & & & & & & & & & & & & & & & & & \\
\hline$\Xi$ & & & & & 0 & & & & & $*$ & $n$ & & $\infty$ & $\infty$ & & & & $\therefore \vec{\omega}$ & & & $\vec{\omega}$ & & & $|*|$ & & \\
\hline$\underline{\Sigma}$ & & & & & & & & & & & $\infty$ & & & & & & & & $\bar{a}$ & $\vec{n}$ & & & & $*$ & & \\
\hline$\Xi$ & & & & & $x$ & & $\hat{x}$ & & & & $n$ & & $n$ & $\vec{\omega}$ & & & & $\vec{n}$ & is & & & & & $*$ & & \\
\hline$\approx$ & & & & $\star x$ & 8 & & & & × & 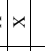 & n & & $n$ & $\vec{\omega}$ & & & & & & & . & & & $\ddot{*} *$ & & \\
\hline$\approx$ & & & & & 5 & & & & & 恙 & . & & $\infty$ & is & & & & & & & 0 & $\approx \infty$ & & & & \\
\hline $\bar{z}$ & & & & & & $x$ & & & & $x$ & $\infty$ & & n & & & $x$ & & & & $x$ & & & & & & \\
\hline 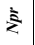 & & & & & \%ั. & & & & & & & & & & & & & & & & & & & $\mid \tilde{*}$ & & \\
\hline 妾 & $\infty$ & & & & & & & & & & 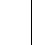 & & & $\infty$ & & & $\therefore$ & & 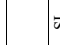 & $\overrightarrow{6}$ & & n & & & & \\
\hline$\ddot{z}$ & & & & & 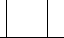 & & & & & & 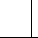 & & & 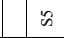 & & & & & & & & & & & & \\
\hline$\underline{\underline{z}}$ & & $n$ & $\infty$ & & 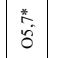 & & $\times$ & & & & $\vec{\omega}$ & & & $\vec{a}$ & & & & & & & & & & & & \\
\hline$\xi$ & & & & & 8 & & & & & 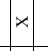 & $\infty$ & & & $\vec{\omega}$ & & & & & & & $\vec{\omega}$ & & & & & \\
\hline 立 & & & & & 8 & & & $\approx$ & & & & & & & & & & & & & & & & & & \\
\hline 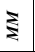 & & & & & & & & & & & & & & & & & & & & & & & & & & \\
\hline$\$$ & & & & & 8 & & $x$ & & $\infty$ & $\star$ & is & & $n$ & $\vec{\omega}$ & & & & & & & $\vec{\omega}$ & & & \begin{tabular}{ll|l}
$*$ & $*$ \\
$\star$ & $*$ \\
$\star$
\end{tabular} & & \\
\hline$\$$ & & & & & & & & & & & & & & is & & & $\therefore$ & & & & $\vec{\infty}$ & & & & & \\
\hline z & & & & & 蓉 & & & & & & & & & & & & & & & & & & & & & \\
\hline 莦 & & & & & $\ddot{\circ}$ & & & & & $*$ & s & & & & & & & & & & & & & $\mid \begin{array}{l}* \\
x\end{array}$ & & \\
\hline 胥 & & & & $>$ & 5 & & & & $\infty$ & & & & & & & & & & & & & & $n$ & $\mid \begin{array}{l}\mid \vec{x} \\
\hat{x}\end{array}$ & & \\
\hline ( & & & & & & & & & & & & & $n$ & $\approx$ & & & & & & & & & & & & \\
\hline$\Xi$ & & & & & $\mid \begin{array}{l}\frac{\hbar}{0} \\
\frac{\partial}{>}\end{array}$ & & & & & & & & & & & & & & & & & & & & & \\
\hline छ & & & & & 范 & & & & & & $\vec{\omega}$ & & & $\vec{\omega}$ & $\sum_{a}^{\infty}$ & & & & & & & & & & & \\
\hline$\Sigma$ & & $\infty$ & & $\times$ & $0 x$ & & $\mid *$ & & & $4 * 2$ & $\vec{n}$ & & & \begin{tabular}{|l|l|}
$*$ & $\infty$
\end{tabular} & & & & $\vec{\omega}$ & & & $\infty$ & & & $\ddot{x} \mid \propto 1$ & & \\
\hline$\approx$ & & & & & 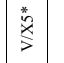 & & & & & & & & & & & & & & & & & & & & & \\
\hline ₹ & & $\infty$ & & $x>$ & $\delta x$ & $x$ & $\times$ & & & $4 \times$ & $n$ & $x$ & & $\vec{\omega} \mid$ & & & & के & $\vec{\sigma}$ & & 0 & & & $\star x$ & $\stackrel{*}{*}$ & $\infty$ \\
\hline ₹ & & & & & ${ }_{0}^{*}$ & & & & & & & & & & & & & & & & & & & & & \\
\hline$=$ & - & $\sim$ & $m$ & + & $\begin{array}{ll}0 & -1\end{array}$ & \begin{tabular}{|l|} 
\\
\end{tabular} & & 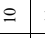 & $=\simeq$ & $\simeq$ & \pm & 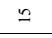 & $\underline{0}$ & $=\simeq$ & $\stackrel{9}{-1}$ & $\therefore$ & $\bar{\nabla} 8$ & $\pi \approx$ & 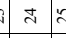 & $\therefore$ & $\bar{a}$ & 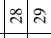 & 8 & $\bar{m}$ & 8 & 黑 \\
\hline 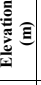 & 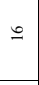 & $\cong$ & $=$ & $\neq \approx$ & $\nexists$ & $\underset{\sim}{\stackrel{0}{*}}$ & & F & 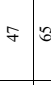 & $\approx$ & 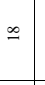 & ते & $F$ & \begin{tabular}{l|l|l|}
$\infty$ & $\infty$
\end{tabular} & $\mathscr{8}$ & $\infty$ & & $=\mathrm{m}$ & $\subseteq-$ & -0 & r & 0 in & $\stackrel{\infty}{\infty}$ & $\Rightarrow=$ & $\approx$ & - \\
\hline 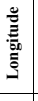 & $\begin{array}{l}\stackrel{\rho}{\hat{\phi}} \\
\bar{\phi} \\
\bar{\phi}\end{array}$ & 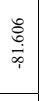 & $\begin{array}{l}\text { : } \\
\dot{\vec{\phi}} \\
\end{array}$ & 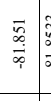 & 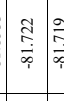 & 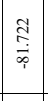 & & 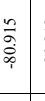 & 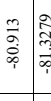 & 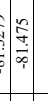 & $\underset{\substack{\vec{p} \\
\underset{p}{f}}}{\underset{f}{f}}$ & 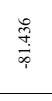 & 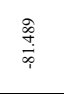 & 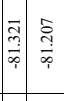 & 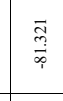 & 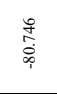 & & 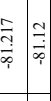 & 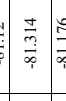 & 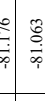 & 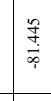 & 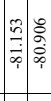 & 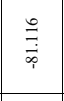 & 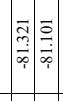 & 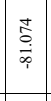 & $\frac{\frac{n}{p}}{\frac{p_{p}}{p_{p}}}$ \\
\hline 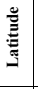 & $\underset{\sim}{\cong}$ & 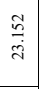 & $\frac{\pi}{\pi}$ & $\begin{array}{ll}f \\
c \\
c\end{array}$ & 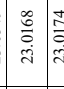 & 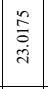 & & 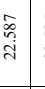 & 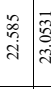 & 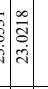 & ్ֻ & 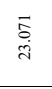 & $\begin{array}{c}\text { o. } \\
\substack{0 \\
\text { in }}\end{array}$ & 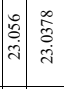 & 范 & $\frac{8}{\mathrm{~d}}$ & & त્নે స్ন & 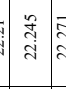 & 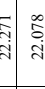 & $\mid$ & 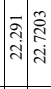 & $\begin{array}{l}0 \\
\stackrel{0}{0} \\
\text { d. }\end{array}$ & 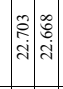 & 总 & $\pi$ \\
\hline 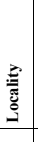 & 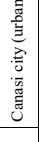 & 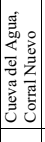 & & | & 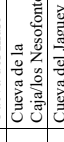 & & & & & & & & 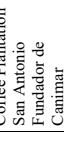 & & 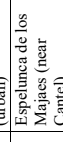 & & 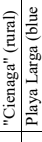 & 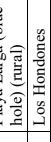 & 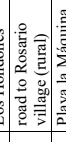 & 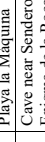 & & & 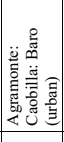 & 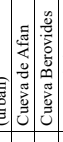 & 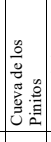 & 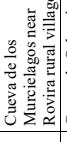 \\
\hline 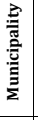 & & & & & 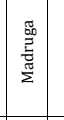 & & & 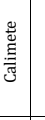 & 量 & & & & & & & 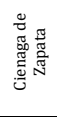 & & & & & & & 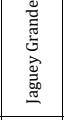 & & & \\
\hline 槖 & 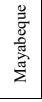 & & & & & & & 急 & & & & & & & & & & & & & & & & & & \\
\hline
\end{tabular}




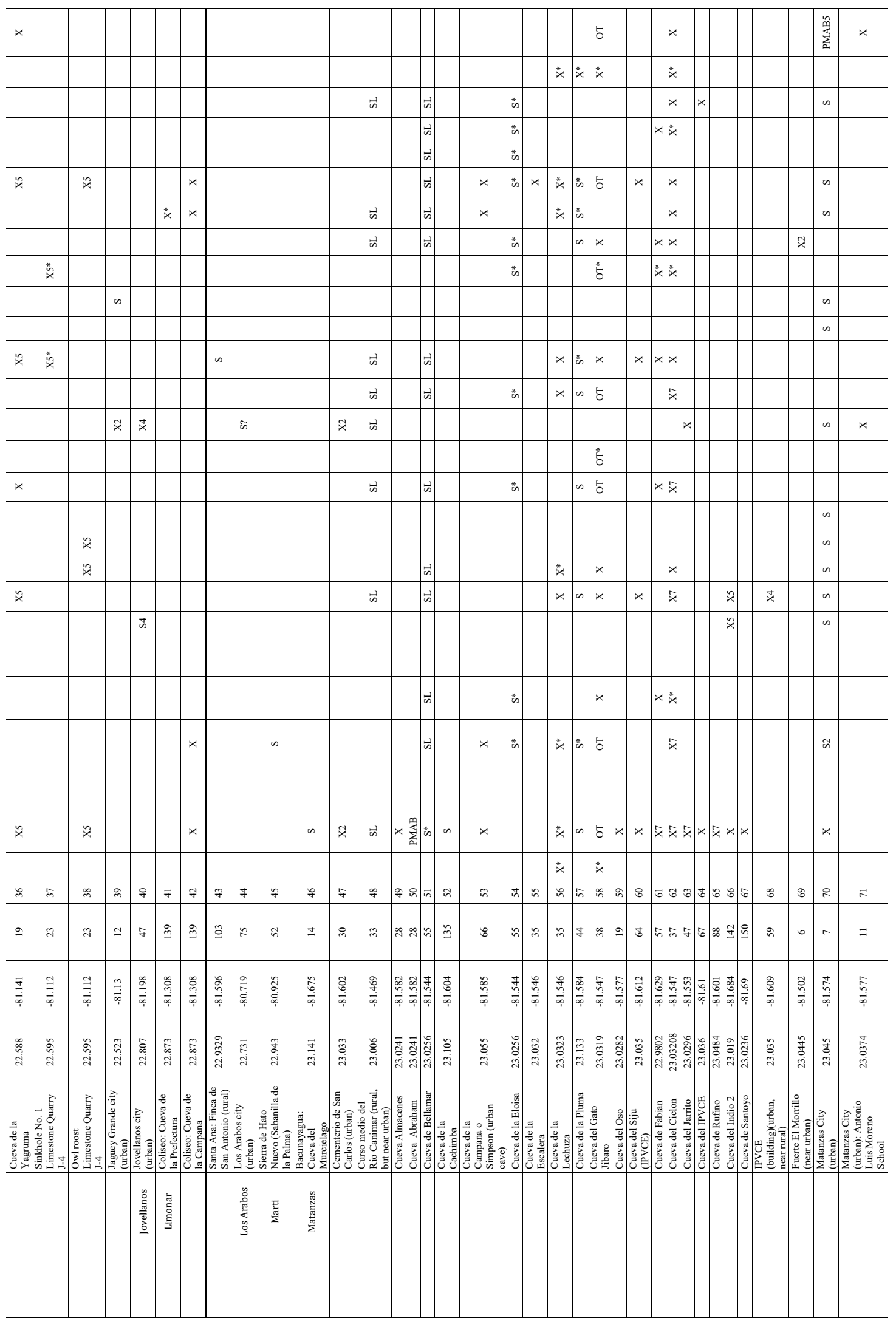




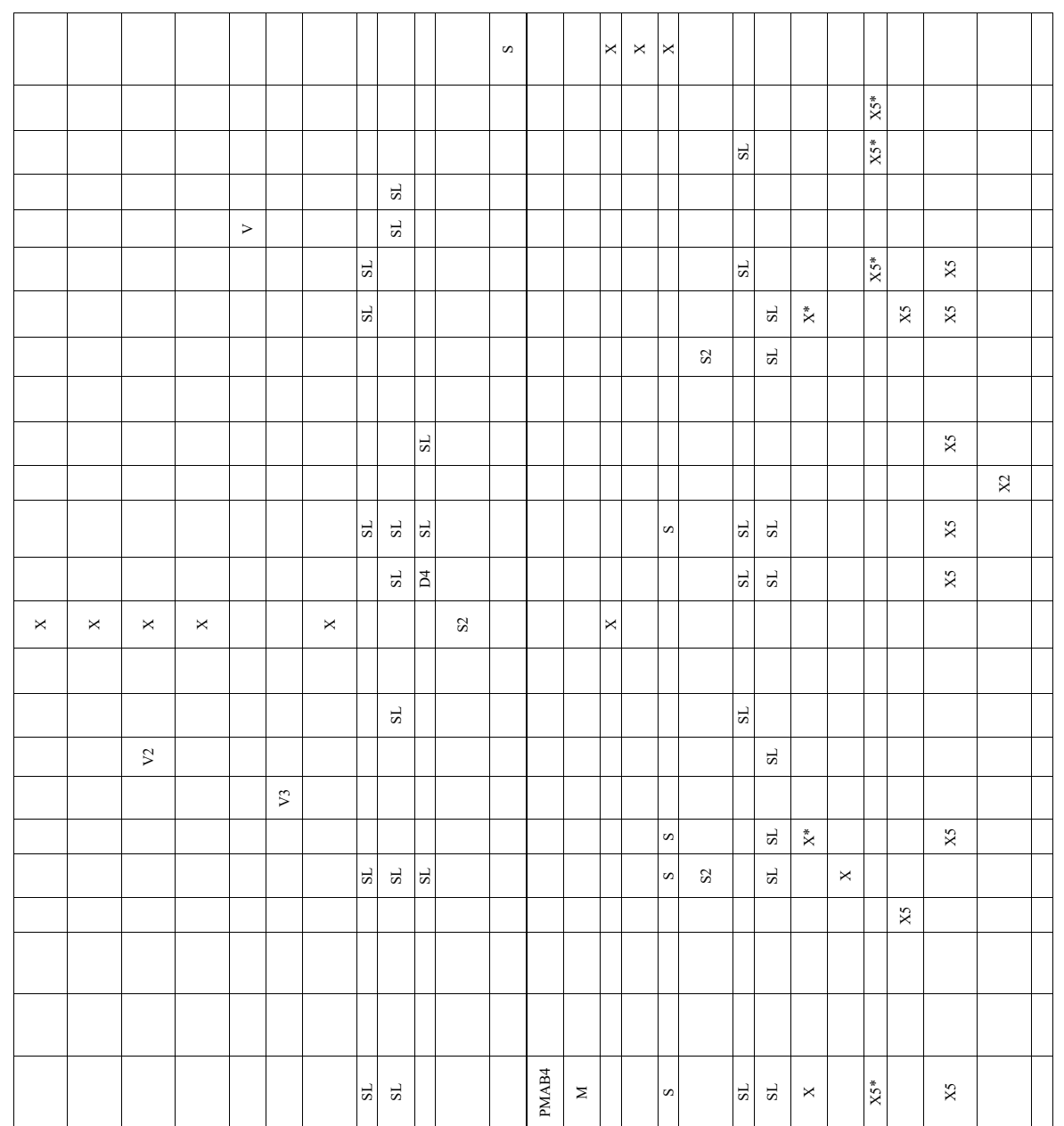

专

कृ चु च

ते क्षे

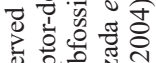

造语

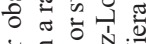

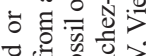

过记

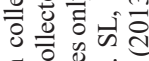

范范

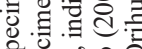

के $* 00$

वृ के च्च

为

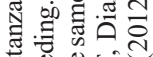

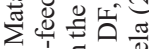

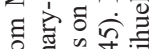

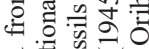

踏

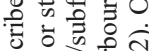

边

$\Rightarrow$ 象

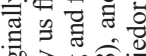

क्षे

y

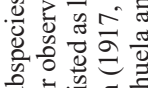

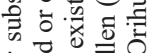

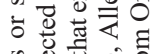

渮

की

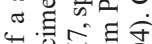

o क्ये

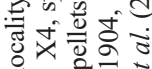

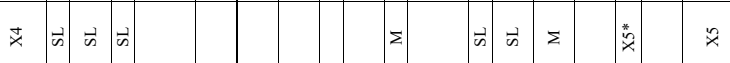

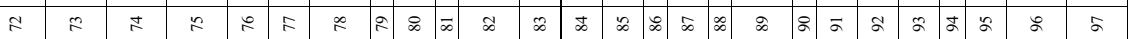

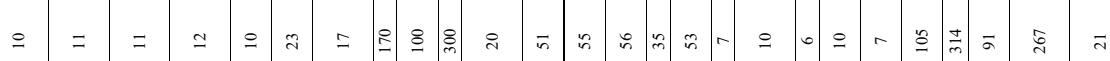

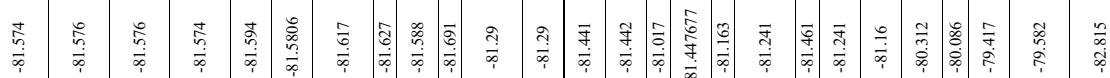

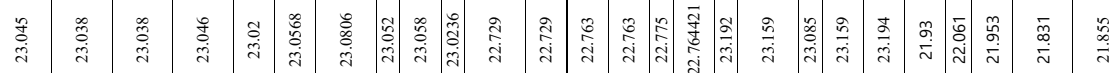

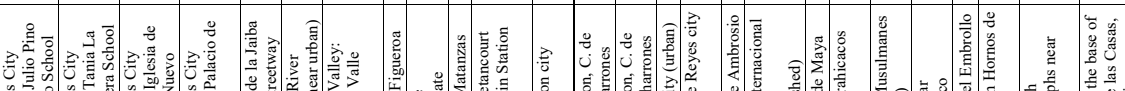

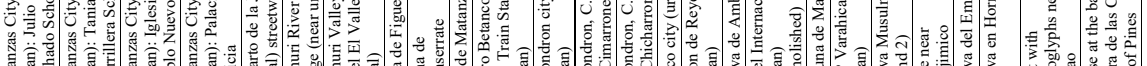

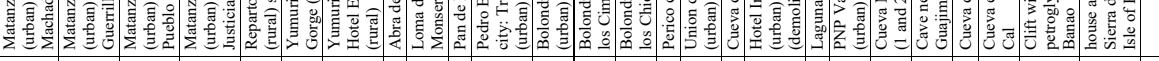

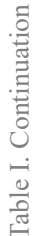

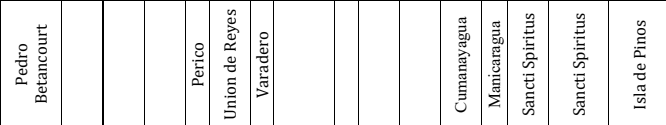

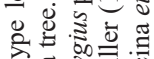

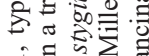

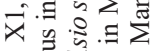

Dे

त्ठ

ঊ

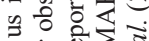

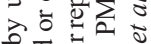

렁혀웡

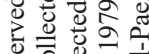

官卷

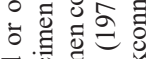

ठู

过司证

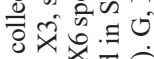

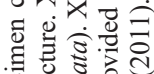

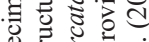

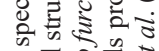

x 雪总

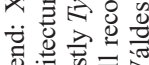

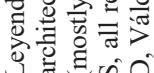




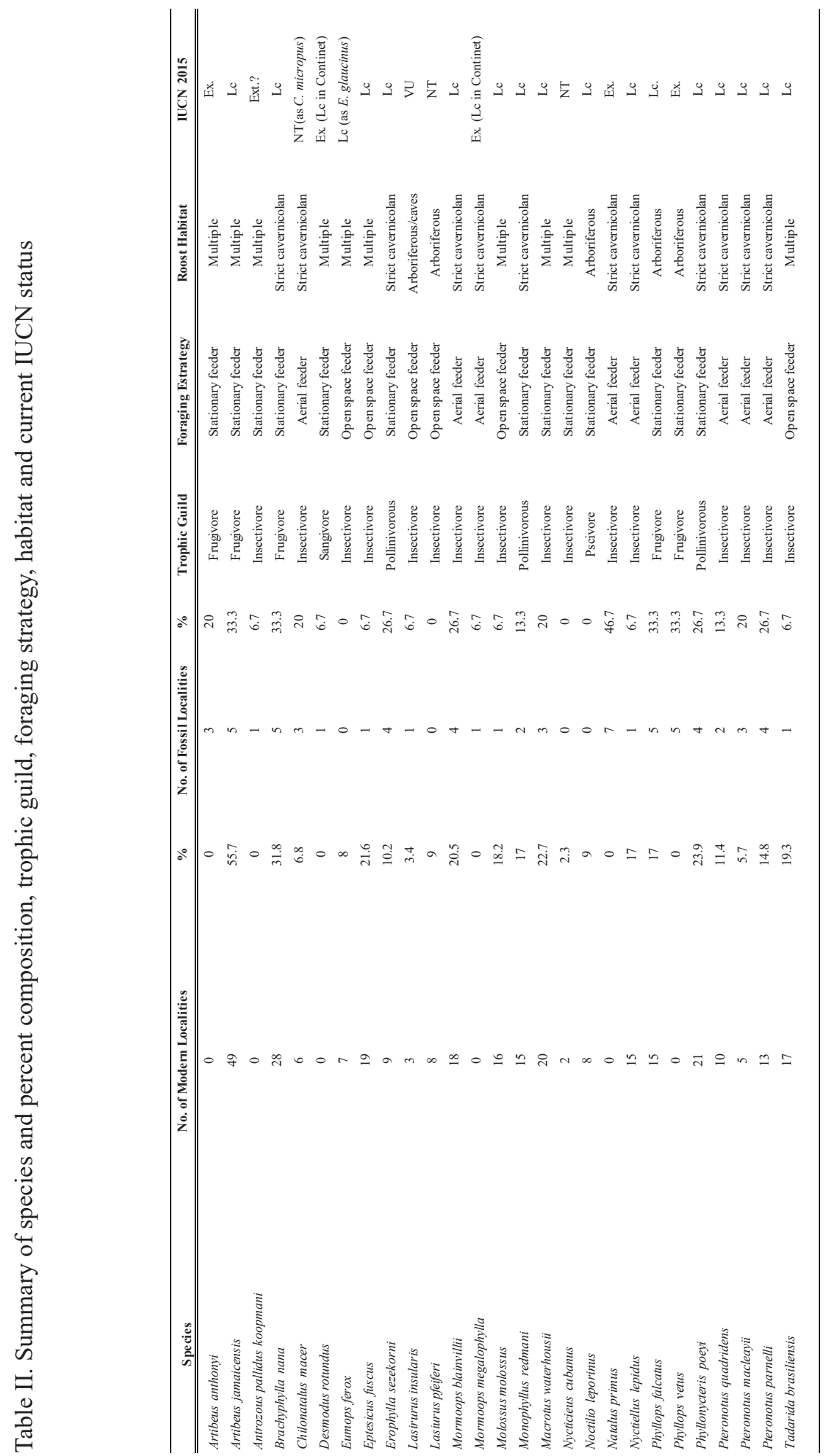




\section{DISCUSSION}

The phyllostomid Artibeus jamaicensis was by far the most common species, collected or observed in 49 localities, representing over $55 \%$ of all collection stations (Table II). Other species occurred at collecting stations as follows: Brachyphylla nana (31.8\%), Phyllonycteris poeyi (23.9\%), Macrotus waterhousii (22.7\%), Eptesicus fuscus (21.6\%) and Mormoops blainvillii (20.5\%). However, the abundance of Tadarida brasiliensis (19.3\%) and Molossus molossus (18.2\%), especially in architectural structures near urban settings, is also noteworthy. Artibeus jamaicensis, Brachyphylla nana, and Phyllonycteris poeyi are also the most frequent bats in the diet of Tyto furcata (Silva Taboada, 1979; Vilato et al., 2002; Hernández-Muñoz \& Mancina, 2011), likely because of their large size and stationary feeding behavior. At the turn of the last century, an expedition was led by William Palmer who summarized that Artibeus jamaicensis, Tadarida brasiliensis, and Eptesicus fuscus were the most common of all Cuban bats (Miller, 1904). This condition similarly persists to date, including also Phyllonycteris poeyi and Macrotus waterhousiii (Silva Taboada, 1979).

The species Artibeus jamaicensis, Brachyphylla nana, Mormoops blainvillii, Monophyllus redmani, Pteronotus parnellii, Phyllonycteris poeyi, and Tadarida brasiliensis have been recorded in booth the live and fossil states in the same localities, suggesting a continuous presence in those areas at least since the middle Holocene (Orihuela et al., in ed.). The rarest living species in our study were Koopman's pallid bat (Antrozous koopmani) with a rarity index of $<2$, the Cuban evening bat (Nycticeius cubanus) with 2.3 and the Cuban yellow bat (Lasiurus insularis) with 3.4.

Nycticeius cubanus is considered a rare species and has not been found in the region's fossil record (Silva Taboada, 1979). However, fossil/subfossil remains of Lasiurus insularis, Phyllops vetus and the critically endangered Antrozous koopmani and Natalus primus suggest a wider distribution for these species in the recent past than at present. Koopmans's pallid bat (Antrozous koopmani) is currently known from a handful of fossil and subfossil remains resulting from raptor-derived pellets, plus only three living specimens captured during the early and middle $20^{\text {th }}$ century (Orr \& Silva Taboada, 1960; Silva Taboada, 1976, 1979; Mancina \& García Rivera, 2005; Hernández-Muñoz \& Mancina, 2011). The Cuban greater funnel-eared bat (Natalus primus) is currently known from a single living population in the extreme west of the island, but its fossil record supports a much wider distribution throughout the Greater Antilles in the past (Tejedor et al., 2004, 2005; Tejedor, 2011).

In the case of the lasiurins their distribution in the archipelago is wide despite their rarity. These taxa have been considered as rare since the late XIX century (Gundlach, 1868). Today, the Cuban lasiurines are known from a handful of records and collecting localities (Silva Taboada, 1979; García \& Mancina, 2011). The Cuban yellow bat (Lasiurus insularis), one of Cuba's rarest bats (Silva Taboada, 1979), is known from less than a dozen collection localities and several fossil/subfossil remains, including "fresh" (i.e., post-Columbian) raptor-derived pellet deposits (Silva Taboada, 1979; Orihuela, 2010; Mancina, 2012; Borroto-Páez \& Mancina, 2017). New specimens, captured or from modern owl pellets, had not been collected for over 40 years (Silva Taboada, 1979; García \& Mancina, 2011; Mancina, 2012; Borroto-Páez \& Mancina, 2017). However, an additional record of Lasiurus insularis and another of Lasiurus pfeifferi since Silva Taboada (1979), but not included in Mancina (2012), García and Mancina (2011), Balseiro (2011) or Sánchez-Lozada et al. (2018), were reported by Viera (2004). Aguayo and Howell Rivero, (1954) reported Lasiurus insularis from Matanzas city (loc. 70) and Orihuela (2010) 
reported it from fossil material at Cueva de la Caja (loc. 6; Orihuela et al., submitted) while Viera (2004) reported a fresh carcass fallen from trees in the Yumurí River Gorge in 1998 (loc. 77; Fig. 3A), in a rural setting near the city of Matanzas. A modern specimen collected in an owl roost at the J4 limestone quarry (loc. 37), captured and deposited in a fresh pellet by a Tyto furcata between 2013 and 2014, is additionally reported here.

The vegetation in the area surrounding the rural Matanzas locality is very depauperated and almost entirely modified for agriculture. Like in the above-mentioned localities, this one is outside a $20 \mathrm{~km}$ radius from any setting with Thrinax-like palms, which Lasiurus insularis prefers for roosting (Silva Taboada, 1979). As discussed in Orihuela (2010), the occurrence in caves, other than raptor-derived cave deposits, may indicate the facultative occasional use of other vegetation types, or caves, as happens with similar lasiurines in North America (De Beaufort, 1934; Quay \& Miller, 1955; Myers, 1960; Grady \& Olson, 2006). Distribution data are obscured in cases of raptor-derived pellet deposits because, owls, for example, can easily carry specimens captured over a dozen km from the roost locality (Dodson \& Wexlar, 1979; Andrews, 1990; Kusmer, 1990). It seems that the rarity of the species may be due to its solitary and arboreal lifestyle, but with an otherwise wide distribution in the Matanzas region and the rest of the island (Table I-II; see Silva Taboada, 1976, 1979).

A similar case occurs for Lasiurus pfeifferi and Chilonatalus macer, both considered rare species by Silva Taboada (1979). Lasiurus pfeifferi had been previously reported from Cárdenas city (loc. 17), from an unknown locality at Zapata Swamp (loc. 20), and from Matanzas city (loc. 70) by Silva Taboada (1979). Viera (2004) had reported an additional fresh carcass at the doorstep of the Pueblo Nuevo church in Matanzas city (loc. 74) Two additional specimens, one from Hicacos Peninsula (Varadero, loc. 91), and one from the rural village of Santo Tómas in the Zapata Swamp (loc. 26) were recently added to the regional list by Sánchez-Lozada et al. (2018), who considered that L. pfeifferi is not as uncommon as previously thought; an opinion we support. These reports have been further confirmed by the Cubabat research group.

Until recently, Chilonatalus macer was known in the province only from fossil and subfossil material found in owl pellet cave deposits (Silva Taboada, 1979; Viera, 2004; Orihuela, 2010). Live specimens have been captured from the rural village of Cocodrilo in the Zapata Swamp and from Cueva Fabian (loc. 61), western Matanzas, in 1998 by one of us (RV). Several other localities have been added since then (13, 16, and 50 in Tejedor, 2011, and Sánchez-Lozano et al. 2018). Additional subfossil-modern remains are now reported from very fresh owl pellet deposits at Cueva del Ciclón (loc. 62) and Gato Jíbaro (loc. 57) where C. macer seems to have been sympatric with Natalus primus until the Amerindian interval of the Late Holocene (authors unp. Data; Orihuela et al., forthcoming). Several fresh specimens of both species have collected at the last two localities.

Among the new occurrences provided by Viera (2004), including the first neontological record of the Cuban evening bat (Nycticeius cubanus) for the Sierra de Caballos in the Isle of Pines, he also recorded Nyctinomops macrotis in 1998 from Palenque Hill, where Cueva de la Caja is located (Viera, 2004). However, we consider this last record erroneous and remove it from the region's bat record because the supposed specimen was not preserved or photographed in detail, thus the identification is now unverifiable. It can be considered questionable due to the similarity of this taxon with Eumops ferox (Silva Taboada, 1979). This species is known from several specimens throughout the island, including fossils and subfossils (Koopman \& Ruibal, 1955; Woloszyn \& Silva Taboada, 1977; Silva Taboada, 1979). Although it is not considered rare, it is relatively scarce (Silva Taboada, 1979; Mancina, 2012). 
Among the extinct species (including those Cuban fossil forms we consider synonymous with extant relatives), Peter's ghost-faced bat (Mormoops megalophylla) and the common vampire bat (Desmodus rotundus) are the rarest in the region, and the two rarest bats of the Cuban fossil record. Their remains have been collected a handful of times in several localities through central-western Cuba (Silva Taboada, 1979; Jiménez et al., 2005; Suárez, 2005; Viera \& Orihuela, 2006; Orihuela, 2010, 2011). In the case of Mormoops megalophylla, new specimens have not been reported since 2012 (Orihuela \& Tejedor, 2012), but more specimens of Desmodus rotundus, yet unreported, are known today from Mayabeque. The extinct short-faced phyllostomid Artibeus anthonyi was the third rarest, recorded only from three localities (5, 55, and 57) in the region of Matanzas. By comparison, Natalus primus was the most common species in all the fossil record, known from seven $(47 \%)$ of the 15 paleontological localities listed here. The three remaining extinct species absent in our study are known only from their type locality (Balseiro, 2011).

Altogether, the fossil and subfossil remains of the extinct, rare and scarce taxa support a wider distribution within the island and a much more recent extinction (Jiménez et al., 2005; Orihuela, 2010; Orihuela \& Tejedor, 2012; Orihuela et al., forthcoming) than were previously recognized. This is also true for the extinct fig-eating bat Phyllops vetus of which we record fossil remains for the first time in Matanzas and Villa Clara. Phyllops vetus had been reported for third in the archipelago in 1992 (Dolón Rodríguez, 1992), but since these results were not published, but presented at a symposium without providing measurements or ilustrations, nor the specimens deposited in a museum collection for further verification, and giving the similarity for the extant Phyllops falcatus, we consider this record uncomfirmable at best.

It is worth mentioning that there is no apparent faunal turnover between the Holocene fossils/subfossils and the modern record of bats in Matanzas; instead there area selective extinctions of a few species and survival of the rest of the known faunal assemblage. At the same time, several of the extinct or extirpated species (A. anthonyi, M. megalophylla, P. vetus) have sister (syntopic) taxa still inhabiting the region, likely occupying similar ecological niches.

From this and other previous studies, it is evident that owl pellet accumulations play a unique role in revealing the distributions of cryptic and solitary species that are otherwise hard to collect. This is especially true for A. koopmani, L. insularis, L. pfeifferi and E. ferox (Silva Taboada, 1976; Hernández-Muñoz \& Mancina, 2011; Vilato et al., 2002). Several other large species with localized flight are relatively common prey of Tyto furcata and Asio stygius, as aforementioned, whereas the small insectivorous bats are less common prey for owls likely because of their higher and faster flight (Silva Taboada, 1979; Jiménez et al., 2005; Hernández-Muñoz \& Mancina, 2011). These records can also help assess anatomical changes in local bat populations, which can be attributed to geographical or chronoclinal variation. For instance,smaller than average Monophyllus redmani, and larger than average Artibeus jamaicensis and Brachyphylla nana have been noted from several deposits in Cueva de la Caja (Orihuela 2010; Orihuela unp. data). Moreover, fossil humeri of Macrotus waterhousii collected from Sinkhole No. 1 Limestone Quarry J4 were significantly smaller in greatest length $(\mathrm{p}>0.05)$ than those of the modern population, a temporal variation previously observed by Silva Taboada (1974) and Jiménez et al. (2005).

The addition of raptor-derived records to diversity inventories provides a unique picture of the bat community in a region, but this surveying method is not without bias. Often, only part of the pellet accumulations is preserved. The presence of certain species may be caused by predator preference and the inclusion of allochthonous species. But even so, pellets can help provide a snapshot of former microvertebrate communities that rarely is integrated to 
the fossil record (Jiménez et al., 2005; author's unpub. data). Because owls show generational fidelity for the same roostings and nesting grounds, some of the sites are active for several thousand years, thus generating unique deposits that can help to understand local faunal and climatic changes through time (Suárez \& Díaz-Franco, 2003; Orihuela et al., in ed). In that sense, pellet accumulation provides a spatial and temporal average, which may combine more than one ecosystem and bat community, and thus record changes of fauna or prey selection/availability. Thus, pellets provide a time-averaged local record that can complement local inventories.

It is likely that for these reasons, in terms of faunal richness and diversity, caves and raptor-derived cave deposits provided the most abundant records, followed only by urban setting records. Apparently, even species that are considered scarce or rare, such a Lasiurus insularis, L. pfeifferi, Chilonatalus macer, Eumops ferox and Phyllops falcatus, can be found near or within urbanized areas. And in many other cases, remains of these bats can be transported by raptors into their roosts from nearby environments.

Many species seem to have adapted and likely benefited from human alteration and urbanization. In Matanzas and other parts of Cuba, the molossids Molossus molossus, Eptesicus fuscus and Tadarida brasiliensis have adapted well to roosting in human-made architectural structures (Silva Taboada, 1979). The city lights attract insects, which in turn are easily preyed upon by these insectivores. We have even observed a Noctilio leporinus catching insects at a light pole in Sabana del Mar, in the Dominican Republic (JO unp. obsv., Nov. 2004; Fig. 4). This phenomenon has been documented for this species in Cuba and Hispaniola (Silva Taboada, 1979; Rodríguez-Duran \& Christenson, 2012; Núñez-Novas et al., 2019), and for other bat species in other parts of the world (Perini et al., 2003; Haupt et al., 2006), and may be beneficial not only to molossids and vespertilionids but also the smaller Cuban natalids. Agricultural fields and fruit plantations, or fruit-bearing arborization in cities, are also attractive to the fruit and pollinivorous bats Artibeus jamaicensis, Brachyphylla nana, Phyllops falcatus, and Phyllonycteris poeyi for instance, which are often observed foraging in urban settings. In that sense, urbanized and agricultural areas do not appear to limit generalist species, but provide a beneficial corridor between rural and natural environments, for several groups.

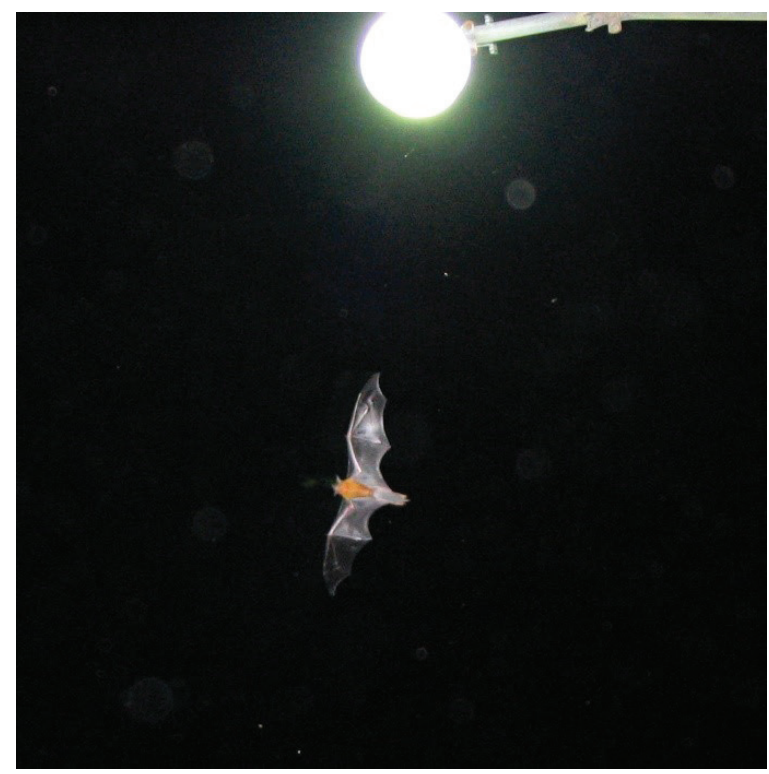

Figure 5. Noctilio leporinus observed preying on insects under a streetlamp at the town of Sabana de la Mar, near Samaná bay, Dominican Republic, November 2004. 


\section{Importance of regional distribution in the conservation of Cuban bats}

The region of Matanzas has played an important role in the history of bat research, specially the taxonomy and systematics of Cuban bats. Six species, including three endemics, were originally described by Johannes Gundlach and Wilhelm Peters during the mid- $19^{\text {th }}$ century from type specimens collected by Gundlach in Cárdenas city and Fundador de Canímar plantation, all in the region of Matanzas (Silva Taboada, 1976; loc. 16 here). Moreover, some of the first fossil bats reported from Cuba were based on specimens collected in caves from Matanzas (Allen, 1917, 1918; Anthony, 1919).

Of the living species recorded for the Cuban archipelago, 21 (80.7\%) representing all six bat families known in Cuba, inhabit the province of Matanzas (Fig. 1, Table I-II). A reason for the high diversity of bats in this region may be that Cuba does not possess major geographical or vegetation barriers such as high mountains or wide deserts. Instead, the island is characterized by its low-lying landscape, with hills that are oriented in parallel to the axis of the archipelago and rarely surpass $400 \mathrm{~m}$ in elevation (Acevedo González, 1992). As a result, bat distribution in Cuba is highly homogeneous, with similar numbers of species found in all other of Cuba's 15 provinces.

The low topography and complex geological history of the archipelago have likely contributed to the biogeography of Cuban bats, especially during the Quaternary. Most species have widespread distributions (wider still during most of the Holocene and up to the recent past, as the fossil record suggests), and only a few species have ranges restricted by the availability of specific vegetation (e.g., Nyctinomops laticuadata and Lasiurus insularis, which prefer savannah biomes with palms of the genus Thrinax sp. and Copernicia sp. Silva Taboada, 1979). Lasiurus pfeifferi seems to be less selective in diurnal roosts, but preferring the foliage of broad-leaved trees (Silva Taboada, 1979). Others, such as Mormopterus minutus, which originally preferred roosting in the palm Copernicia gigas (= vespertilium; Henderson et al., 1995) have recently adapted to roosting in architectural structures (Silva Taboada, 1979).

Sixteen species or $60 \%$ of Cuban bats use caves in one way or another, and of these 10 (37 \%) are strict cave dwellers, preferring rooms with especially hot microclimates or "hot caves" (Silva Taboada, 1976, 1979; Morgan, 2001; Mancina et al., 2004). In that sense, the geological history of the archipelago and its proclivity towards cave formation in limestone karst terrain bears importance in Cuban bat past and present distribution (Silva Taboada, 1979).

Matanzas harbors today the most extensive subaerial karst region of the entire Cuban archipelago, a potentially cave-rich region $\sim 65,500 \mathrm{~km}^{2}$ wide (Acevedo González, 1992). Probably, no other province in Cuba has more caves available for cave-roosting bats today than the region of Matanzas. However, at the end of the Ice Age, 11,500 years ago, Pinar del Río and the Gulf of Batabanó, south of the western half of Cuba had the largest potential in the availability of caves for bat roosting anywhere in the Cuban archipelago, competing in size only with the Bahama bank. Once the ice of the last glacial maximum melted with the warmer temperatures of the Holocene epoch, sea level rose and inundated some of the Cuban ancient karst plains, drowning about $\sim 13,300 \mathrm{~km}^{2}$ of latent cave-rich territory (Nuñez et al., 1984, 1989), essential for bat life in the island, and likely culling the territory of several species. Many have postulated this as an important reason for the disappearance of several cave-dwelling bat species (Morgan \& Woods, 1986; Morgan, 2001; Dávalos \& Russell, 2012, Soto-Centeno et al., 2015). However, fossil and subfossil material indicate the survival of most bat species well into the late pre-Columbian interval of the late Holocene, even after all the fluctuations of the late Pleistocene and the Holocene, including several thousands of years of human colonization of the archipelago (Orihuela, 2010; Orihuela \& Tejedor, 2012; Borroto-Páez \& Mancina, 2017; Orihuela et al., in ed.). 
Deforestation and other forms of human environmental destruction and disturbance since European colonization seem to have been a pivotal factor in the survival of Cuban bats (Borroto-Páez \& Mancina, 2017). Although a significant part of the original forest vegetation has been eliminated or replaced by agricultural and secondary vegetation since the mid-late $18^{\text {th }}$ century (Marrero, 1972, 1976; Monzote, 2008; Borroto-Páez \& Mancina, 2017), the abundance of caves and few preserved patches of forest seem to have aided in maintaining the overall distribution and bat diversity observed in most of the Cuban archipelago. This is true for some species, but such deforestation as a form of habitat elimination was likely detrimental to species such as the lasiurines, Antrozous koopmani and Natalus primus (Silva Taboada, 1979).

\section{CONCLUSIONS}

Our compilation of bat distribution records in the region of Matanzas, plus several sparse records from the Isle of Pines and the central region of the main island of Cuba, incorporating neontological and paleontological data, indicate that bat diversity in the region has been generally stable but some species had a much wider range since the middle Holocene that lasted until very recently. This is especially true for locally extinct species Natalus primus and Antrozous koopmani, which seem to have persisted in the region until the very late Holocene and likely up to the post-Columbian/colonial period. The globally extinct Artibeus anthonyi and Phyllops vetus, reported for the first time in the province, support a slightly higher phyllostomid diversity in the area, and a wider range within the archipelago.

However, the knowledge of the bat fauna in most areas of the Matanzas region, as in others of the archipelago, is limited to a few records, generally lacking detailed and prolonged inventories. One of these areas is the Zapata Swamp, from which only a handful of records exists (Silva Taboada, 1979; Díaz-Franco, 2004). Inventories are especially needed from areas of high urbanization touristic development such as in developing areas of Varahicacos and central parts of the province, including urban populations, to better understand the situation of bat communities there and their response to human alterations. Accelerating changes in environmental degradation or modifications driven by human techno-economic endeavors are known to affect species with restricted ranges outside of nature reserves or national parks.

Our records, taken with other published accounts, indicate that urban centers are an attractive hub to some species which seem to be adapting and benefiting from human alteration. Some of Cuba's rarest or scarce bats, such as Lasiurus insularis, L. pfeifferi and Chilonatalus macer have been collected directly in urban areas, suggesting that urban centers can act as corridors between rural and preserved areas (e.g., Matanzas city, the Valle de Yumurí, and the high forests of the Pan de Matanzas-Palenque hills).

The data we presented here are relevant to the understanding and overall estimation of Cuban bat diversity and distribution. Furthermore, they can help provide sensible-location and temporal-spatial distribution data to complement capture-only inventories within the island, crucial to environmental and faunal conservation in the archipelago.

\section{ACKNOWLEDGMENTS}

Many thanks are due to Leonel Pérez Orozco, Candido Santana, Jorge Garcell, Osvaldo Jiménez and Joel Monzón for their help with field research and logistics during the last two decades. To Adrian Tejedor, Gilberto Silva Taboada and Osvaldo Jiménez enlightening discussion on Cuban bats and extinction. Thanks are also due to Nick Czaplewski who corrected the first versions of the manuscript, to Carlos Suriel, and one anonymous referee provided suggestions and corrections that helped better this paper. 


\section{LITERATURE CITED}

Acevedo González, M. 1992. Geografía física de Cuba. Editorial Pueblo y Educación, La Habana, Cuba, 389 pp.

Aguayo, C. G. 1950. Observación sobre algunos mamíferos cubanos extinguidos. Boletín de Historia Natural de la Sociedad Felipe Poey, 1: 121-134.

Aguayo, C. G., \& L. Howell Rivero. 1954. Sinopsis de los mamíferos cubanos. Circulación del Museo y Biblioteca Zoológicas, La Habana: 1283-1324.

Allen, G. M. 1911. Mammals of the West Indies. Bulletin of the Museum of Comparative Zoology, 40: 173-263.

Allen, G. M. 1917. New fossil mammals from Cuba. Bulletin of the Museum of Comparative Zoology, 61: 3-12.

Allen, G. M. 1918. Fossil mammals from Cuba. Bulletin of the Museum of Comparative Zoology, 62: $133-148$.

Andrews, P. 1990. Owls, Caves and Fossils. University of Chicago Press, Chicago, 231 pp.

Anthony, H. E. 1917. New rabbit and a new bat from Neotropical regions. Bulletin of the American Museum of Natural History, 37: 335-337.

Anthony, H. E. 1919. Mammals collected in Eastern Cuba. Bulletin of the American Museum of Natural History, 41: 625-643.

Balseiro, F. 2011. Los Murciélagos extintos. Pp. 171-177, In: R. Borroto Páez, \& C. A. Mancina (Eds.) Mamíferos en Cuba. UPC Print, Vaasa, Finland.

Barbour, T. 1945. A Naturalist in Cuba. Little Brown and Co., Boston, 317 pp.

Barrios, D., \& C. A. Mancina. 2017. Dendrocereus nudiflorus (Cactacea): pasado, presente y futuro de un anacronismo en peligro de extinción según modelos de nicho climático. Revista del Jardin Botanico Nacional, 38: 119-132.

Borroto-Páez, R., \& C. A. Mancina. 2017. Biodiversity and conservation of Cuban mammals: past, present, and invasive species. Journal of Mammalogy, 98 (4): 964-985.

Chapman, F. M. 1892. Notes on the birds and mammals observed near Trinidad, Cuba, with remarks on the origin of West Indian bird life. Bulletin of the American Museum of Natural History, 4: 279-330.

Dávalos, L. M., \& A. L. Russell. 2012. Deglaciation explains bat extinction in the Caribbean. Ecology and Evolution, 2 (12): 3045-3051.

De Beaufort, C. F. 1934. Dasypterus intermedius H. Allen in Cuba. Journal of Mammalogy, 15: 316.

Díaz-Franco, S. 2004. Análisis de la extinción de algunos mamíferos Cubanos, sobre la base de evidencias paleonotológicas y arqueológicas. Revista biológica, 18 (2): 147-154. 
Díaz-Franco, S. 2005. Mammals, p. 110-111. In A. Kirkconnell, D. F. Stotz, and J. M. Shopland (Eds), Cuba: Península de Zapata. Rapid Biological Inventories 07, The Field Museum, Chicago.

Dodson, P., \& D. Wexlar. 1979. Taphonomic investigation of owl pellets. Paleobiology, 5 (3): $275-284$.

Dolón Rodríguez, E. M. 1992. Hallazgo de dos craneos de Stenoderma vetun [=Phyllops vetus] (Orden Chiroptera, Taxon fosil) Tercer reporte para Cuba. Resumenes del II Congreso Espeleológico de Latinoamerica y del Caribe. Viñales, Pinar del Río, Cuba, septiembre de 1992.

García Rivera, L., \& C. A. Mancina. 2011. Murciélagos insectívoros, p: 140-165. In R. Borroto-Páez, and C. A. Mancina (eds), Mamiferos en Cuba. UPC, Vasa, Finland.

Garrido, O. H. 1980. Los vertebrados terrestres de la Península de Zapata. Poeyana, 203: 1-49.

González Alonso, H., L. Rodríguez, A. Rodríguez, C. A. Mancina, \& I. Ramos (Eds.). 2012. Libro Rojo de los Vertebrados de Cuba. Editorial Academia, La Habana, 304 pp.

Grady, F. V., \& S. L. Olson. 2006. Fossil bats from Quaternary deposits on Bermuda (Chiroptera: Vespertilionidae). Journal of Mammalogy, 87 (1): 148-152.

Gundlach, J. 1868. Revista y catálogo de los mamiferos cubanos. Repertorio Fisico-Natural de la Isla de Cuba, II: 40-56. Imprenta de la viuda de Barcina y Comp. La Habana.

Haupt, M., S. Menzler, \& S. Schmidt. 2006. Flexibility of habitat use in Eptesicus nilssonii: Does the species profit from Anthopologically altered habitats? Journal of Mammalogy, 87 (2): 351-361.

Henderson, A., G. Galeano, \& R. Bernal. 1995. Field Guide to the Pals of the Americas. Princeton University Press, Princeton, 502 pp.

Hernández-Muñoz, A., \& C. A. Mancina. 2011. La dieta de la lechuza (Tyto alba) (Aves: Strigiformes) en hábitats naturales y antropogénicos de la región central de Cuba. Revista mexicana de biodiversidad, 82 (1): 217-226.

Instituto de Planificación Física. 1989. Nuevo Atlas Nacional de Cuba. Instituto de Geografía de la Academia de Ciencias de Cuba y el Instituto Cubano de Geodesia y Cartografía La Habana, Cuba, 288 pp.

Jiménez, O., M. M. Condis, \& E. G. Cancio. 2005. Vertebrados post-glaciales en un residuario fósil de Tyto alba Scopoli (Aves: Tytonidae) en el occidente de Cuba. Revista Mexicana de Mastozoología, 9 (1): 85-112.

Kirkiconnell-Paez, A., D. F. Stotz, \& J. M. Shopland (Eds). 2005. Cuba: Península de Zapata. Rapid Biological Inventories Report 07. The Field Museum, Chicago, 96 pp.

Koopman, K. F., \& R. Ruibal. 1955. Cave-fossil vertebrates from Camagüey, Cuba. Breviora, 46: 1-8.

Kusmer, K. D. 1990. Taphonomy of owl pellet deposition. Journal of Paleontology, 64 (4): 629-637. 
Longino, J. T., \& R. K. Colwell. 1997. Biodiversity assessment using structured inventory: capturing the ant faun of a tropical rain forest. Ecological Applications, 7 (4): 1263-1277.

Mancina, C. A. 2011. Introducción a los murciélagos, p. 123-133. In R. Borroto-Páez, and C. A. Mancina, (Eds), Mamíferos en Cuba. UPC Print, Vaasa, Finland.

Mancina, C. A. 2012. Mamíferos, p. 268-291. In H. González Alonso et al. (Eds), Libro Rojo de los Vertebrados de Cuba. Editorial Academia, La Habana, Cuba.

Mancina, C. A., A. Hernández Marrero, \& A. D. Álvarez. 2004. Catálogo de los murciélagos (Mammalia: Chiroptera) depositados en el Instituto de Ecología y Sistemática, La Habana, Cuba. Instituto de Ecología y Sistemática, La Habana (Cuba). Poeyana, 492: 14-33.

Mancina, C. A., \& L. García Rivera. 2011. Murciélagos fitófagos, p. 134-147. In R. Borroto-Páez, and C. A. Mancina (Eds), Mamíferos en Cuba. UPC Print, Vaasa, Finland.

Marrero, L. 1972. Cuba: Economía y Sociedad Vol. 1. Editorial Playor, S. A., Madrid, 309 pp.

Marrero, L. 1976. Cuba: Economía y Sociedad Vol. 5. Editorial Playor, S. A., Madrid, 227 pp.

Miller, G. S. 1904. Notes on the bats collected by William Palmer in Cuba. Proceedings of the U. S. National Museum, 27 (1359): 337-348.

Monzote, R. F. 2008. From Rainforest to Cane Field in Cuba: An Environmental History since 1492. University of North Carolina Press, North Carolina, 384 pp.

Morgan, G. S. 2001. Patterns of extinction in West Indian bats, p. 369-407. In C. A. Woods, and F. E. Sergile (Eds), Biogeography of the West Indies: Patters and Perspectives. CRC Press, Boca Raton, 608 pp.

Morgan, G. S., \& C. A. Woods. 1986. Extinction and zoogeography of the West Indian landmammals. Biology Journal of the Linnaean Society, 28: 167-203.

Myers, R. F. 1960. Lasiurus from Missouri caves. Journal of Mammalogy, 41 (1): 114-117.

Nuñez Jiménez, A., N. Viña Bayes, M. Acevedo González, M. Iturralde Vinent, \& A. Graña González. 1984. Cuevas y Carsos. Editorial Militar, La Habana, Cuba, 431 pp.

Nuñez Jiménez, A. 1989 [1990]. Medio Siglo Explorando a Cuba (Tomo 1-2). Imprenta Central de las FAR, La Habana, Cuba, 560 pp.

Núñez-Novas, M. S., R. Guzmán-Pérez, \& A. Contreras-Puigbó. 2019. Guía de identificación de los murciélagos de La Española y anotaciones sobre las familias y especies. Novitates Caribaea, 13: 39-63.

Orihuela, J. 2010 [2012]. Late Holocene Fauna from a Cave Deposit in Western Cuba: post Columbian occurrence of the Vampire Bat Desmodus rotundus (Phyllostomidae: Desmodontinae). Caribbean Journal of Science, 46: 297-312.

Orihuela, J. 2011. Skull variation of the vampire bat Desmodus rotundus (Chiroptera: Phyllostomidae): Taxonomic implications for the Cuban fossil vampire bat Desmodus puntajudensis. Chiroptera Neotropical, 17 (1): 963-976. 
Orihuela, J. 2013. Fossil Cuban crow Corvus cf. nasicus from a Late Quaternary cave deposit in northern Matanzas, Cuba. Journal of Caribbean Ornithology, 26: 12-16.

Orihuela, J. \& A. Tejedor. 2012. Peter's ghost-faced bat Mormoops megalophylla (Chiroptera: Mormoopidae) from a pre-Columbian archeological deposit in Cuba. Acta Chiropterologica, 14: 63-72.

Orihuela, J., L. Pérez Orozco, R. J. L. Álvarez, A. Viera \& C. Santana (in ed.). Late Holocene land vertebrate fauna from Cueva de los Nesofontes, Western Cuba: last appearance dates and paleoecology. Submitted to Paleontologia Electronica.

Orihuela, J., L. W. Viñola, O. Hernández de Lara, A. Mychajliw, O. Jiménez, L. Lorenzo, \& A. Soto-Centeno. (in ed.). The role of human son Greater Antillean land vertebrate extintions: new insights from Cuba.

Orr, R. T. \& G. Silva Taboada Taboada. 1960. A new species of bat of the genus Antrozous from Cuba. Proceedings of the Biological Society of Washington, 73: 83-86.

Perini, F. A., V. C. Távares, \& C. M. D. Nascimento. 2003. Bats from the city of Belo Horizonte, Minas Gerais, southeastern Brazil. Chiroptera Neotropical, 9: 169-172.

Quay, W. B., \& J. S. Miller. 1955. Occurrence of the Red bat, Lasiurus borealis in caves. Journal of Mammalogy, 36 (6): 454-455.

Rodríguez-Durán, A., \& K. Christenson. 2012. Breviario sobre los murciélagos de Puerto Rico, La Española e Islas Vírgenes, Universidad Interamericana de Puerto Rico, 104 pp.

Sánchez-Lozada, M., H.V. Rodríguez, H. M. D. Perdomo, J. M. González, A. H. de la Cruz Mora, A. L. Muñoz, A. Loyola, T. M. Espinosa, A. Rodríguez-Cabrera, A.V. Bertucciolli, \& C. A., Mancina González. 2018. Datos de distribución de murciélagos en Cuba: un acercamiento a través de inventarios biológicos rápidos. Poeyana, 507: 76-81.

Silva-Taboada, G. 1974. Fossil Chiroptera from cave deposits in central Cuba, with a description of two new species (genera Pteronotus and Mormoops) and the first West Indian record of Mormoops megalophylla. Acta Zoologica Cracoviensia, 19: 33-73.

Silva Taboada, G. 1976. Historia y actualización taxonómica de algunas especies antillanas de murciélagos de los géneros Pteronotus, Brachyphylla, Lasiurus, y Antrozous. Poeyana, 153: 1-24.

Silva Taboada, G. 1979. Los Murciélagos de Cuba. La Habana, Cuba, Editorial Academia, $423 \mathrm{pp}$.

Silva Taboada, G., \& H. Vela Rodríguez. 2009. Actualización taxonómica y distribucional de los murciélagos de Cuba (1). El Explorador, 26 de mayo.

Simmons, N. B. 2005. Order Chiroptera. In D. E. Wilson and D. M. Reeder (Eds). Mammal species of the world: A Taxonomic and Geographic reference, $3^{r d}$. ed. Washington D.C. Smithsonian Institution Press, 2,142 pp.

Soto-Centeno, J. A., M. O’Brien, \& N. B. Simmons. 2015. The importance of late Quaternary climate change and karst on distributions of Caribbean mormoopid bats. American Museum Novitates, 3847: 1-32. 
Suárez W. 2005. Taxonomic Status of the Cuban Vampire Bat (Chiroptera: Phyllostomidae: Desmodontinae: Desmodus). Caribbean Journal of Science, 41 (4): 761-767.

Suárez, W., \& S. Díaz-Franco. 2003. A new fossil bat (Chiroptera: Phyllostomidae) from a Quaternary cave deposit in Cuba. Caribbean Journal of Science, 39 (3): 371-377.

Tejedor, A. 2011. Systematics of funnel-eared bats (Chiroptera: Natalidae). Bulletin of the American Museum of Natural History, 353: 1-140.

Tejedor, A., G. Silva Taboada, \& D. R. Hernández. 2004. Discovery of extant Natalus major (Chiroptera: Natalidae) in Cuba. Mammalian Biology, 69: 153-162.

Tejedor, A., V. D. Tavares, \& G. Silva-Taboada. 2005. A revision of extant Greater Antillean bats of the genus Natalus. American Museum Novitates, 3493: 1-22.

Valdés, D. B., L. R. González-Torres, \& A. P. Bejerano. 2011. Biología de la polinización de Leptocereus scopulophilus (Cactaceae) en el Pan de Matanzas, Cuba. Revista del Jardín Botánico Nacional, 32-33: 163-168.

Viera, R. A. 2004. Aportes a la quiropterofauna nacional. 1861 Revista de Espeleología y Arqueología, 5 (1): 21-23.

Viera Muñoz, R. A., \& J. Orihuela. 2006. Importante hallazgo de murciélago vampiro. Mundo Interior, 2 (1): 13-15.

Vilato, R., D. M. Barroso, A. D. Freyre, \& J. M. Townsend. 2002. Importancia alimentaria en la dieta de la lechuza Tyto alba furcata (Aves: Strigiformes) en la Ciudad de Camagüey, Cuba. El Pitirre, 15: 61-64.

Woloszyn, B. W., \& G. Silva Taboada. 1977. Nueva especie fósil de Artibeus (Mammalia: Chiroptera) de Cuba, y tipificación preliminar de los depósitos fosilíferos Cubanos contentivos de mamíferos terrestres. Poeyana, 161: 1-17.

[Recibido: 01 de julio, 2019. Aceptado para publicación: 28 de octubre, 2019] 